\title{
Agarose Gel Microcapsules Enable Easy-to-prepare, Picolitre-scale Single-cell Genomics, Yielding Near- complete Genome Sequences
}

Hiroyoshi Aoki ( $\nabla$ haoki@riken.jp )

RIKEN Center for Advanced Photonics

Yuki Masahiro

RIKEN BioResource Research Center

Michiru Shimizu

RIKEN BioResource Research Center

Yuichi Hongoh

Tokyo Institute of Technology

Moriya Ohkuma

RIKEN BioResource Research Center

Yutaka Yamagata

RIKEN Center for Advanced Photonics

\section{Research Article}

Keywords:

Posted Date: February 3rd, 2022

DOI: https://doi.org/10.21203/rs.3.rs-147972/v2

License: (9) (i) This work is licensed under a Creative Commons Attribution 4.0 International License.

Read Full License 
1 Agarose gel microcapsules enable easy-to-prepare, picolitre-scale single-cell

2 genomics, yielding near-complete genome sequences

3

4

5 Hiroyoshi Aoki ${ }^{1,4^{*}}$, Masahiro Yuki ${ }^{2,4}$, Michiru Shimizu ${ }^{2}$, Yuichi Hongoh ${ }^{2,3,5}$,

6 Moriya Ohkuma ${ }^{2,5^{*}}$, and Yutaka Yamagata ${ }^{1,5}$

7

81 Ultrahigh Precision Optics Technology Team, Advanced Photonics Technology

9 Group, RIKEN Center for Advanced Photonics, Wako, Saitama, Japan.

102 Japan Collection of Microorganisms (JCM), RIKEN BioResource Research Center,

11 Tsukuba, Ibaraki, Japan.

123 School of Life Science and Technology, Tokyo Institute of Technology, Tokyo,

13 Japan.

144 These authors contributed equally to this work.

155 These authors contributed equally to this work.

$18 *$ Corresponding authors

19 Hiroyoshi Aoki. Address: 3-1, Hirosawa, Wako, Saitama, 351-0198, Tel.: +81-48-467-

20 9315. Fax.: +81-48-462-4655. E-mail: haoki@riken.jp.

21 Moriya Ohkuma. Address: 3-1-1, Koyadai, Tsukuba, Ibaraki, 305-0074, Japan. Tel.:

22 +81-29-829-9101. Fax.: +81-29-836-9561. E-mail: mohkuma@riken.jp. 


\section{Abstract}

25 A novel type of agarose gel microcapsule (AGM), consisting of an alginate picolitre sol

26 core and an agarose gel shell, was developed to obtain high-quality single-cell amplified

27 genomic DNA of bacteria. The AGM is easy to prepare in a stable emulsion with oil of

28 water-equivalent density which prevents AGM aggregation, with only standard

29 laboratory equipment. Single cells from a pure culture of Escherichia coli, a mock

30 community comprising 15 strains of human gut bacteria, and a termite gut bacterial

31 community were encapsulated within AGMs, and their genomic DNA samples were

32 prepared with massively parallel amplifications in a tube. The genome sequencing did

33 not need second-round amplification, and showed an average genome completeness that

34 was much higher than that obtained using a conventional amplification method in

35 microlitre scale, regardless of the genomic guanine-cytosine contents. Our novel method

36 using the AGM allows many researchers to perform single-cell genomics easily and

37 effectively, and can accelerate the genome analysis of yet-uncultured microorganisms. 


\section{Introduction}

41 Microorganisms are ubiquitously distributed in diverse environments. They are often

42 associated with other organisms, and play important roles in ecosystems. However, the

43 majority of microbial species are difficult to culture with conventional methods and are

44 called yet-uncultured microorganisms ${ }^{1}$. In the past two decades, they have been

45 extensively studied using culture-independent methods such as amplicon sequencing

46 analysis of small subunit ribosomal RNA (SSU rRNA) genes and shotgun sequencing

47 analysis of metagenomes. Metagenomics is a powerful tool for investigating the

48 ecological and physiological functions of microbiota based on their encoded genetic

49 repertories. The recent development of the computational binning of metagenomic

50 fragments into respective microbial taxonomic assemblies has reinforced the utility of

51 metagenomics ${ }^{2,3}$. However, computational binning based on the sequence composition,

52 homology to database sequences, and sequence read coverage of each fragment can

53 often fail to discriminate genomes of closely related (sub)species and to correctly sort

54 genomic regions exhibiting distinct features from others, such as rRNA genes,

55 prophages, and plasmids ${ }^{4,5}$. Furthermore, a massive sequencing effort is required to

56 obtain the genome sequences of minor species in the microbial community ${ }^{3}$. 
58 physically isolated single cells ${ }^{6}$, has been used as an alternative or complementary

59 method to reveal the metabolic capacity of yet-uncultured microorganisms ${ }^{1,7}$. Single-

60 cell isolation can be achieved in many cases by using technologies such as fluorescence-

61 activated cell sorting (FACS $)^{1}$, micromanipulators ${ }^{8}$, microfluidic devices $^{9,10}$, and

62 encapsulation in water-in-oil droplets ${ }^{11}$. Although various methods for whole genome

63 amplification have been developed ${ }^{12}$, multiple displacement amplification (MDA) using

64 phi29 DNA polymerase with random primers is most widely used due to its relative

65 simplicity, reliability, and applicability ${ }^{6,13}$. However, MDA inherently accompanies

66 extreme amplification bias among genome regions ${ }^{1}$, which has been the major technical

67 limitation in single-cell genomics. For example, the amplification bias caused lower

68 average completeness of single-cell amplified genomes (SAGs) (47\%) than that of

69 metagenome-assembled genomes (MAGs) (93\%) in a study of marine samples,

70 although use of different coverage regions between SAG and MAG helped to obtain

71 entire genome sequences ${ }^{14}$. Additionally, the high guanine-cytosine (GC) content of

72 genomic DNA tends to increase the amplification bias ${ }^{1,15}$. It has been reported that

73 MDA in a small reaction volume, for example, when using a microchannel chamber (60

$74 \mathrm{~nL})^{10}$, nano-litre microwells $(12 \mathrm{~nL})^{16}$, virtual microfluidics (MDA in gel sheet, 60 
$\mathrm{nL})^{17}$, digital droplets generated by a microchannel $(9.5-240 \mathrm{pL})^{18-20}$, and gel bead ${ }^{21}$,

76 can suppress amplification bias. Additionally, suppression of amplification bias using

77 droplet MDA has been proved effective also for metagenomics: the droplet MDA more

78 accurately presented microorganism composition than conventional microlitre scale

79 bulk $\mathrm{MDA}^{22}$. However, these microfabrication techniques are not available for many

80 microbiologists, and the amount of the amplification product is often too small for the

81 direct genome sequencing; therefore, a second-round MDA is often required, which

82 ultimately enhances the amplification bias $^{23}$.

83 To accelerate study using the single-cell genomics of yet-uncultured

84 microorganisms, it is important to develop a simple method that prepares massive small

85 reaction vessels using commercially available reagents, disposable plasticware, and

86 equipment. For MDA reaction vessels, a microchannel can massively generate single-

87 cell encapsulating water-in-oil droplets ${ }^{11}$. However, MDA reagents are needed to be

88 sequentially introduced into the droplets in the microchannel, which complexifies the

89 microchannel structure. In contrast, single-cell encapsulating gel beads are more easily

90 prepared by gelating cell-containing solated gel droplets ${ }^{21,23,24}$. The MDA of the single-

91 cell genome in the bead can be done only by exchanging reagents through gel. 
92 However, the amount of MDA product in the gel matrix is generally insufficient for

93 next generation DNA sequencing.

94 A hollow-core hydrogel microcapsule ${ }^{25}$, which consists of a hydrogel shell and a

95 sol core, is expected to be an efficient vessel for single-cell MDA. Many hollow-core

96 hydrogel microcapsules have been reported to be useful for cell cultivations and cell

97 transplantations: for example, pairs of a crosslinked polyethylene glycol (PEG) gel shell

98 and a PEG sol core ${ }^{26}$, an alginate and poly-L-lysine complex shell and an alginate sol

$99 \operatorname{core}^{27}$, and an agarose gel shell and an alginate sacrifice core ${ }^{28}$. Recently, MDA in a

100 hollow-core hydrogel microcapsule was used for discriminating single bacterial cells

101 based on fluorescent in situ hybridization targeting a protein-coding gene ${ }^{29}$. The

102 microcapsule was prepared using simple emulsification gelation and consisted of a

103 chemically-crosslinked soluble polyacrylamide gel core, an agarose gel middle layer,

104 and a PCR primer-conjugated polyacrylamide gel shell. After genomic DNA from

105 single Escherichia coli was amplified in the gel core using MDA, the target gene in the

106 MDA product was further amplified in the microcapsule using emulsion PCR after

107 solation of the core and the middle layer. However, the MDA product from single-cell

108 genomic DNA, which was amplified in a picolitre-scale hollow core in a microcapsule,

109 has not been fully evaluated for single-cell genomics. 
110 Here, we introduce a novel method for single-cell genomics, using a newly

111 developed AGM consisting of an agarose gel shell and an alginate sol hollow-core,

112 which enables single-cell isolation and picolitre-scale MDA (Fig. 1a). The fabrication

113 process of the AGM was optimised here for easier preparation using inexpensive

114 equipment and reagents, such as a vortex mixer, centrifuges, and commercially

115 available reagents without hazardous chemical crosslinker (Fig. 1b), although the size

116 uniformity of AGM is sacrificed compared to the methods using microfabrication

117 techniques ${ }^{29,30}$. Thus, this novel type of AGM can be easily prepared and is scalable for

118 many microbiologists. By using AGM, a single bacterial cell can be encapsulated in its

119 picolitre-scale sol core and then subjected to MDA just by exchanging solutions

120 (designated here 'MDA-in-AGM'). We demonstrate that MDA-in-AGM enables

121 massively parallel single-cell genomics with much improved genome completeness,

122 compared to a conventional method using FACS and microlitre-scale MDA.

123

124 Results

125 Preparation of AGMs containing single bacterial cells. Alginate gel cores

126 containing bacterial cells were prepared using the emulsification/internal gelation

127 method $^{30}$ (Fig. 1b). Briefly, a mixture of alginate solution, bacterial cells, and $\mathrm{CaCO}_{3}$ 
nanoparticles was emulsified with isostearyl alcohol (ISA). The resulting microdroplets

129 were gelated with calcium ions that were released from $\mathrm{CaCO}_{3}$ nanoparticles by the

130 addition of acetic acid to the water-in-oil emulsion. The concentration of bacterial cells

131 was optimised using serial dilutions so that one alginate microdroplet contained one or

132 no bacterial cell. The resulting alginate gel cores were washed sequentially with diethyl

133 ether, 1-butanol, and Tris- $\mathrm{HCl}(\mathrm{pH} 7.5)$ buffer.

134 Agarose solution (SeaPlaque, Lonza; final 2\%) was added to the alginate gel

135 cores suspended in Tris- $\mathrm{HCl}(\mathrm{pH} 7.5)$ at $35^{\circ} \mathrm{C}$, and the mixture was emulsified with

$1360.25 \%(\mathrm{v} / \mathrm{v})$ Span 80 in polyglyceryl-6 octacaprylate (PGO) at $35^{\circ} \mathrm{C}$ (Fig. 1b). The use

137 of PGO with a density of $0.997 \mathrm{~g} / \mathrm{mL}$, which is equivalent to that of water and AGMs

$138(\sim 1.0 \mathrm{~g} / \mathrm{mL})$, enabled the formation of a stable water-in-oil emulsion during the gradual

139 gelation of agarose by cooling at $4^{\circ} \mathrm{C}$. In addition, PGO improved the AGM preparation

140 process by preventing the aggregation of AGMs and increasing their diameter and yield

141 (Supplementary Fig. 1). After PGO was removed by mixing with diethyl ether and

142 further with 1-butanol, the alginate gel cores were solated with a one-tenth volume of

$1430.5 \mathrm{M}$ ethylenediaminetetraacetic acid (EDTA). The solation of alginate cores in AGMs

144 was confirmed based on the core dissolution into the buffer after dissolving the agarose

145 shells (Supplementary Fig. 2). The alginate sol core is expected to provide an 
146 appropriate space for the MDA reaction, which was shown by the much greater yields

147 of amplified DNA than those with an alginate gel core or agarose gel bead, as described

148 below. The AGMs were washed with Tris-EDTA buffer ( $\mathrm{pH}$ 7.4) to remove excess

149 EDTA, and then suspended in a one-half volume of Tris-EDTA buffer and stored at

$150 \quad 4^{\circ} \mathrm{C}$

151 Escherichia coli DH5 $\alpha$ was used as a model microorganism. E. coli cells were

152 added at a concentration of $3 \times 10^{8}$ cells $\mathrm{mL}^{-1}$ alginate- $\mathrm{CaCO}_{3}$ solution and were

153 encapsulated in $5.64 \pm 1.72 \times 10^{5} \mathrm{AGMs}($ mean $\pm \mathrm{SD})$ in three independent

154 experiments, which corresponded to $12.5 \% \pm 5.4 \%$ of the total AGMs. Of the AGMs

155 containing E. coli cells, approximately $94 \%$ harboured a single cell in the core

156 (Supplementary Table 1). In the three independent experiments, the diameters of the

157 prepared AGMs were $39.6 \pm 22.2 \mu \mathrm{m}, 51.4 \pm 13.3 \mu \mathrm{m}$ and $49.3 \pm 19.6 \mu \mathrm{m}$

158 (Supplementary Fig. 3a). AGM diameters and core volumes showed normal

159 distributions and log-normal distributions, respectively (Supplementary Figs. 3b and 4).

160 The average of medians of AGM core volumes in the three experiments was $15.1 \pm 6.3$

$161 \mathrm{pL}(\mathrm{n}=3)$. 
163 MDA of single-cell genomes within AGMs. AGMs (in $50 \mu \mathrm{L}$ suspension) were

164 washed with sterile water and collected by centrifugation. The lysis of bacterial cells

165 and denaturation of double-stranded DNA within the AGMs were carried out at room

166 temperature for 30 min with $50 \mu \mathrm{L}$ of an alkaline solution, Buffer D2 of the REPLI-g

167 UltraFast Mini Kit (Qiagen). Denaturation was stopped by neutralisation with the

168 addition of the same volume of Stop solution from the kit, and the supernatant was then

169 removed by centrifugation. Reaction Buffer $(93.5 \mu \mathrm{L})$ containing $11 \mu \mathrm{L}$ of REPLI-g

170 UltraFast DNA Polymerase from the kit was added, and the resulting mixture was

171 incubated at $30^{\circ} \mathrm{C}$ for $3 \mathrm{~h}$. The reaction was stopped with one-tenth volume of $0.5 \mathrm{M}$

172 EDTA, and the AGMs were washed three times with $200 \mu \mathrm{L}$ of Tris-EDTA buffer. All

173 the reagents used in MDA and the AGM preparation were irradiated with ultraviolet

174 light to degrade any contaminating DNA prior to use.

Among $4.6 \pm 2.9 \times 10^{5}$ AGMs prepared with E. coli cells, $8.9 \% \pm 0.8 \%$

176 exhibited DNA amplification, which corresponded to $77.0 \% \pm 27.2 \%$ of the AGMs

177 containing E. coli cells $(\mathrm{n}=3)$ (Supplementary Table 1). As shown in Fig. 2, amplified

178 DNA filled the alginate sol core, whereas AGMs with alginate gel cores that were

179 prepared without the solation step with EDTA or agarose gel beads containing no

180 alginate core exhibited only limited or no DNA amplification by MDA. 
182 Quality of single-cell genome sequences compared between MDA-in-AGM and

183 a conventional method using FACS-MDA. The quality of SAGs was evaluated and

184 compared with that obtained by a conventional method using a combination of FACS

185 for single-cell isolation and MDA (FACS-MDA) on a microlitre scale. AGMs

186 containing amplified DNA that was stained with SYBR Green I were individually

187 transferred to $0.2 \mathrm{~mL}$ PCR tubes with $29 \mu \mathrm{L}$ of sterile water under an epifluorescence

188 microscope equipped with a TransferMan NK2 micromanipulator (Eppendorf).

189 Amplified DNA was released to water by heating the tubes at $60^{\circ} \mathrm{C}$ for $5 \mathrm{~min}$, and

190 directly used for preparation of sequencing libraries using the QIAseq FX DNA Library

191 Kit (Qiagen). No second round of MDA was necessary to obtain the libraries suitable

192 for genome sequencing. Paired-end sequence reads were generated on the Illumina

193 MiSeq platform with the Reagent Kit V3 (600 cycles), and a fixed number of read pairs

194 were randomly chosen and assembled de novo into contigs using SPAdes 3.13.0 31 after

195 standard quality filtering. Contigs $>1 \mathrm{~kb}$ were used for the subsequent analyses. Several

196 factors describing the genome sequence quality, including completeness and

197 contamination rate, were evaluated using CheckM ${ }^{32}$ and QUAST ${ }^{33}$, and compared

198 between the SAGs obtained using MDA-in-AGM and FACS-MDA, respectively. 
200 obtained by MDA-in-AGM rapidly increased during the sequencing effort, and the

201 average genome completeness reached $93.0 \% \pm 4.7 \%(n=10)$ for assemblies using $9 \times$

$20210^{5}$ read pairs, while it was 33.6\% $\pm 17.4 \%(\mathrm{n}=10)$ in FACS-MDA (Student's $t$-test, $P$

$203<0.01)($ Fig. 3a and Supplementary Table 2). The genome coverage calculated by

204 mapping reads to the reference E. coli DH5 $\alpha$ genome sequence (BOCF01000000) was

$20598.0 \% \pm 1.7 \%$ in MDA-in-AGM $(\mathrm{n}=10)$, which was much higher than $52.0 \% \pm 13.8 \%$

206 in FACS-MDA $(\mathrm{n}=10)$ for $9 \times 10^{5}$ read pairs $(P<0.01)($ Supplementary Table 2$)$. In

207 addition, the amplification bias among genome regions, which is inherent to MDA, was

208 much improved in MDA-in-AGM compared to FACS-MDA (Fig. 3b). The steep

209 decrease of number of contigs, i.e., smooth assembly of contigs, in MDA-in-AGM

210 along the sequencing effort's depth was probably owing to the lower amplification bias

211 (Fig. 3a). Other quality metrics of SAGs using all sequence reads are shown in

212 Supplementary Table 3.

214 Single-cell genome analyses with a mock community and a natural sample. To

215 evaluate the feasibility of MDA-in-AGM in a more realistic sample, we constructed a

216 mock community comprising 15 cultured strains of human gut bacteria. The taxonomy 
217 and compositions of each bacterial strain estimated using 16S rRNA amplicon analysis

218 or metagenome analysis are shown in Supplementary Table 4. SAGs from the mock

219 community were obtained using either MDA-in-AGM or FACS-MDA in the same way

220 as above. Taxonomic compositions of SAGs were similar between MDA-in-AGM and

221 FACS-MDA, both of which successfully recovered SAGs of most strains accounting for

$222>5 \%$ of the mock community except Faecalibacterium prausnitzii (Supplementary

223 Table 4). The overall genome completeness of SAGs with $<5 \%$ contamination was

224 significantly higher in MDA-in-AGM $(68.0 \% \pm 23.3 \%, \mathrm{n}=39)$ than in FACS-MDA

$225(42.4 \% \pm 20.5 \%, \mathrm{n}=36)$ for assemblies using $3 \times 10^{5}$ read pairs $(P<0.01)$

226 (Supplementary Tables 5 and 6). For example, SAGs of Bacteroides thetaiotaomicron

227 and Parabacteroides distasonis exhibited clear differences between MDA-in-AGM and

228 FACS-MDA in the genome completeness, total sequence length, number of contigs, and

229 amplification bias, as seen in the E. coli SAGs (Fig. 3). The SAGs of most other strains

230 also showed similar results (Supplementary Fig. 5).

Most sequence reads were mapped to their respective reference genomes: the

mapping rates were $94.8 \%$ and $97.3 \%$ (median) for SAGs from MDA-in-AGM and

233 FACS-MDA, respectively (Supplementary Table 7). Although the rates of cross 
235 lower than 5\% (Supplementary Table 7). In Ba. thetaiotaomicron SAGs, sequence reads

236 from a $\mathrm{Ba}$. thetaiotaomicron plasmid were also obtained (Supplementary Table 7). Our

237 results indicated that MDA-in-AGM is applicable to various bacterial species and

238 improves genome completeness in both gram-positive and gram-negative bacteria,

239 regardless of their GC content (Fig. 4). As for Fa. prausnitzii, it is possible that the cell

240 lysis process using $\mathrm{KOH}$ was not effective and hence no SAGs were obtained in both

241 MDA-in-AGM and FACS-MDA.

242

SAGs from MDA-in-AGM with $>5 \%$ contamination, which would be caused

243 either by contaminating extracellular DNA or by multiple cells being encapsulated in a

244 single AGM, were binned into each bacterial species using metaWRAP ${ }^{34}$. As a result,

24530 additional SAGs with $<5 \%$ contamination were obtained, and their genome

246 completeness and contamination rates were $73.5 \%$ (median) and $0.9 \%$ (median),

247 respectively (Supplementary Table 8).

248 DNA rearrangement during MDA, i.e., formation of chimeras, can complicate

249 de novo assembly of single-cell genomes ${ }^{35}$. In E. coli SAGs, the rates of chimeric reads

250 were slightly higher in MDA-in-AGMs $(6.28 \% \pm 1.09 \%)$ than in FACS-MDAs $(4.49 \%$

$251 \pm 1.32 \%)($ Welch's $t$-test, $P<0.01$; Supplementary Table 3$)$. In SAGs from the mock 
2545 and 6). These rates of chimeric reads in MDA-in-AGM were similar to or slightly

255 higher than the rate $6.19 \%$ in a previous study using a conventional MDA method for

256 sequencing human haploid genomes ${ }^{36}$.

Finally, we applied MDA-in-AGM to a natural environmental sample. We used

258 the microbiota in the gut of the termite Reticulitermes speratus, which comprises

259 several hundred bacterial species from diverse phyla ${ }^{37}$. The entire guts of five worker

260 termites were removed, and the gut contents were suspended. Then, bacterial cells were

261 collected by centrifugation after digesting the extracellular DNA with DNase I, and the

262 cells were washed with sterile water as described previously ${ }^{38}$. The subsequent

263 procedures were the same as those described above. We analysed 48 SAGs obtained by

264 MDA-in-AGM and found high genome completeness $(78.6 \% \pm 18.2 \%)$ and a low

265 contamination rate $(1.0 \% \pm 1.0 \%)$ (Table 1$)$. The SAGs were taxonomically classified

266 using the Genome Taxonomy Database Tool Kit (GTDB-Tk) ${ }^{39}$ and were found to be

267 affiliated with 13 bacterial classes, including known dominant groups in the termite gut,

268 Spirochaetia, Bacteroidia, Alphaproteobacteria, Betaproteobacteria,

269 Deltaproteobacteria, Epsilonproteobacteria, and Clostridia ${ }^{37}$. In contrast, the genome completeness of SAGs obtained by FACS-MDA was only $37.7 \% \pm 19.0 \%(\mathrm{n}=161)$. 
271 Although a different sequencing method, i.e., a combination of the Nextera XT DNA

272 Library Prep Kit (Illumina) and the Illumina HiSeq 2500 platform (500 cycles), was

273 used for the latter case, the number of reads (bases) used for assembly was much larger

274 on average $(551 \mathrm{Mb} \pm 64 \mathrm{Mb}, \mathrm{n}=161)$ than in the analysis of SAGs obtained by MDA-

275 in-AGM (256 Mb $\pm 49 \mathrm{Mb}, \mathrm{n}=48)$ (Supplementary Table 9).

276 In addition to the 48 SAGs, we analysed 18 SAGs of the class Endomicrobia

277 with small genome sizes $(\sim 1 \mathrm{Mb})^{40}$ obtained by MDA-in-AGM, and $91.2 \% \pm 9.8 \%$

278 genome completeness was obtained. Furthermore, among them, eight SAGs of

279 phylotype Rs-D17 were compared to the complete genome sequences obtained

280 previously ${ }^{40,41}$. The genome coverages calculated by mapping reads onto the two

281 genomovars of Rs-D17 $\left(\mathrm{Ri} 2008^{40}\right.$ and Ti2005 $\left.{ }^{41}\right)$ reached $95.7 \% \pm 1.3 \%$ and $96.7 \% \pm$

$2820.9 \%$, respectively (Supplementary Table 10). The composition of the termite gut

283 community was estimated from SAGs using MDA-in-AGM or 16S rRNA amplicon

284 analysis (Supplementary Table 11). The taxonomic composition of the SAGs showed a

285 high positive correlation $(\mathrm{R}=0.834, P<0.01$; Pearson's correlation coefficient $)$ with

286 that shown by the $16 \mathrm{~S}$ rRNA amplicon analysis, whereas the frequencies of gram-

287 positive bacteria were lower in MDA-in-AGM. 
290 MDA-in-AGM, the massively parallel MDAs in the picolitre-scale AGM cores,

291 drastically improved the genome completeness of SAGs of E. coli, human gut bacteria

292 in a mock community, and bacteria in the termite gut, compared to conventional

293 microlitre scale FACS-MDA. Thus, the method is expected to contribute to revealing

294 the metabolic capacity of yet-uncultured prokaryotes in environments such as animal

295 intestinal tracts, soil, and hydrosphere.

296 It has been reported that the amplification bias in single-cell genomics can be

297 suppressed by reducing the reaction volume of MDA using microfabrication

298 techniques ${ }^{10,16,18,21,24}$. The comparisons of MDA-in-AGM and other MDA methods are

299 shown in Supplementary Table 12. Although the high genome completeness was also

300 achieved in other miniaturised MDA methods, MDA-in-AGM does not require

301 specialised equipment and provides scalable sample preparation.

303 MDA compared to that obtained by MDA in gels (Fig. 2). This is probably attributable

304 to the enhanced diffusivity of amplified DNA in the sol core ${ }^{42}$. MDA-in-AGM therefore

305 does not require a second round of MDA, which is often necessary in other methods 
second-round MDA step resulted in the high genome completeness with suppressed

308 amplification bias and enabled the simpler workflow.

309 The formation of chimeric sequences during MDA can affect the preciseness of

310 the de novo assembly of $\mathrm{SAGs}^{43}$. In the mock community, the rates of chimeric reads

311 were comparable with those in droplet $\mathrm{MDA}^{20}$ and slightly higher than conventional

312 MDA methods (Supplementary Table 12$)^{36}$. Since the formation of chimeras is likely

313 attributable to the branch migration of amplified $\mathrm{DNA}^{35}$, a higher MDA reaction

314 temperature by using thermostable phi29 DNA polymerase ${ }^{15}$ may decrease erroneous

315 hybridization between replicons.

316 Our MDA-in-AGM is applicable to both gram-positive and gram-negative

317 bacteria and also to genomes with high GC content (Fig. 4). Additional advantages of

318 the AGM are its physical stability and permeability for small molecules, which have

319 made buffer exchange and physical handling much easier. Although MDA-in-AGM can

320 be performed without specialised equipment, the size distribution of AGM is difficult to

321 control and the manual picking procedure using a micromanipulator suppresses

322 throughput. To increase the uniformity of AGM and throughput of single-cell genomics

323 using MDA-in-AGM, microchannels for preparation of uniform AGM, high-speed 
324 AGM sorting using FACS, and molecular-barcoding ${ }^{44}$ of SAGs may be adapted,

325 although these approaches reduce the simplicity of the procedure.

326 Although the taxonomic composition of SAGs obtained by MDA-in-AGM was

327 largely consistent with those shown by $16 \mathrm{~S}$ rRNA amplicon and metagenomic analyses,

328 a SAG of dominant Fa. prausnitzii was not recovered in either MDA-in-AGM or

329 FACS-MDA (Supplementary Table 4). Thus, the failure was not specific to MDA-in-

330 AGM but attributable to the MDA procedure. Since similar discrepancies in the

331 taxonomic composition were found in gram-positive bacterial classes in the experiment

332 using the termite gut microbiota (Supplementary Table 11), modification of cell lysis

333 procedure, including an addition of lysozyme, may be required for certain gram-positive

334 bacteria. To optimise such conditions, AGM is also superior because the exchange of

335 solution is easier compared to other methods.

336 Lower average genome completeness of the mock community $(68 \%)$ and the

337 termite gut microbiome (79\%) than that of E. coli $(98 \%)$ (Supplementary Tables 2 and

338 5; Table 1) may also be attributable to the difference in easiness of cell lysis. Low

339 genome completeness of SAGs in certain bacterial lineages and environmental samples,

340 compared to SAGs of cultured E. coli, have been reported in previous studies,

341 irrespective of the MDA methods ${ }^{1,45}$. For example, in droplet single-cell MDA, the 
342 genome completeness of E. coli and soil microbiota were $96.5 \% \pm 2.2 \%(\mathrm{n}=16)$ and

$34352.8 \% \pm 24.3 \%(\mathrm{n}=17)$, respectively ${ }^{20}$. Cell lysis optimisation for different prokaryotic

344 strains and environmental samples will be important also for increasing genome

345 completeness.

346 Recently, when our experiments were on going, MDA using a hollow-core

347 hydrogel microcapsule consisting of a crosslinked PEG gel shell and a dextran sol core,

348 a similar capsule structure to AGM, was reported ${ }^{42}$. Although amplification bias has not

349 been evaluated, single-cell MDA in the core increases the amount of MDA product

350 when compared with MDA in the gel bead. In addition, the shell of a hollow-core

351 hydrogel microcapsule prevents genomic DNA leaking from the core after alkaline

352 denaturation prior to MDA. However, near-ultraviolet (UV) light (365 nm) used for

353 photo chemical crosslinking of the PEG shell damaged cells and DNA due to

354 photooxidation $^{46}$. We selected agarose as the AGM shell because it allows thermally

355 mild reversible gelation, is commercially available, and is stable in MDA reagents,

356 especially alkaline, neutralisation buffer, and EDTA.

357 AGMs or other reaction chambers containing single bacterial cells inevitably

358 produces a proportion of chambers containing multiple bacterial cells $\mathrm{s}^{23}$, and it is

359 difficult to completely eliminate contaminating DNA in our experimental procedures, as 
360 in other previously developed methods ${ }^{1}$. Nevertheless, the application of a computer

361 program for binning metagenomic fragments, such as metaWRAP ${ }^{34}$, enabled us to

362 recover a considerable number of, at least, median-quality SAGs from mini-

363 metagenome bins, which were resulted from multiple cells, by eliminating

364 contaminating sequences (Supplementary Table 8).

365 In recent years, numerous studies analysing MAGs from environmental samples

366 have been reported ${ }^{47}$. Although single-cell genomics has different advantages and

367 potentially increases the quality of research in combination with metagenomics, for

368 example, by revealing strain-level heterogeneity ${ }^{48}$ and by providing information on

369 horizontally transferred genetic components ${ }^{24}$, it is difficult for many microbiologists to

370 perform. MDA-in-AGM is a much easier and less expensive method, and in addition,

371 only a small amount of sample is needed. Thus, this method is also suitable for tiny

372 specimens, such as the intestinal tracts of small insects and protist cells with endo- and

373 ectosymbiotic bacterial communities.

375 Methods

376 Reagents. Ultrapure DNase/RNase-Free Distilled Water (Thermo Fisher Scientific,

377 Waltham, MA) was used for all solutions prepared in-house. Solutions were autoclaved 
at $121^{\circ} \mathrm{C}$ for $15 \mathrm{~min}$ and further decontaminated by $\mathrm{UV}$ radiation at $7.2 \mathrm{~mW} / \mathrm{cm}^{2}$ overnight (12 h) in a laminar flow cabinet. and $\mathrm{pH}$ of the $\mathrm{CaCO}_{3}$ suspension were reduced by washing the particles using three volumes of water. As well as Shiraishi's $\mathrm{CaCO}_{3}$, fine calcium carbonate such as precipitated calcium carbonate BioUltra (approximately 3- $\mu$ m diameter; Sigma, St.

385 Louis, MO) is also used for alginate core gelation. Alginate solution (5\%,w/v) was

386 prepared by dissolving sodium alginate $(80-120 \mathrm{cP}$ at $1 \% \mathrm{w} / \mathrm{v}$, Wako, Osaka, Japan) in

387 water and stored at $4^{\circ} \mathrm{C}$. Agarose solution was prepared by dissolving SeaPlaque (final

$3882 \%, w / v$, Lonza, Basel, Switzerland) in water, and stored at $4^{\circ} \mathrm{C}$. Polyglyceryl-6 octacaprylate (PGO; Nisshin Oillio, Tokyo, Japan), and Tris-EDTA buffer (TE;

390 BioUltra for molecular biology, $\mathrm{pH}$ 7.4, Sigma), and REPLI-g UltraFast Mini Kit

391 (Qiagen, Hilden, Germany) were used as described below.

393 Evaluation of PGO for agarose microdroplet gelation. After agarose solution (2

$394 \mathrm{~mL}$ ) was mixed with PGO or ISA, each containing $0.25 \%$ sorbitan monooleate (Span 395 80; Wako) $(\mathrm{v} / \mathrm{v})\left(20 \mathrm{~mL}, 35^{\circ} \mathrm{C}\right)$, by vortexing and gelated on ice, agarose beads were 
396 recovered from PGO or ISA as described below. Large agarose aggregates were

397 removed from the agarose beads using 300- $\mu \mathrm{m}$ cell strainers (pluriSelect, Leipzig,

398 Germany). The yields and diameters measured with ImageJ (https://imagej.nih.gov/ij/)

399 of the agarose beads were statically analysed with R (https://www.r-project.org/).

400

401 Preparation of Escherichia coli stock culture. After E. coli DH5 $\alpha$ (TaKaRa Bio Inc)

402 from a single colony on LB plate was cultured in two LB $(200 \mathrm{~mL})$ media, Cells of $E$.

403 coli were harvested and washed twice in PBS (20 mL) by centrifugation. The cells were

404 re-suspended in PBS $(10 \mathrm{~mL})$, and their density was determined with a cell-counting

405 chamber. The $E$. coli cells $\left(3.05 \times 10^{10}\right.$ cell $\left./ \mathrm{mL}\right)$ were divided into aliquots in

406 microtubes $(0.5 \mathrm{~mL}$ each $)$ and stored at $-80^{\circ} \mathrm{C}$.

407

408 Preparation of alginate cores containing E. coli cells. Cells of E. coli were

409 encapsulated in alginate cores by the emulsification/internal gelation method ${ }^{30}$ (Fig. 1b).

410 E. coli cells from the stock culture $(10 \mu \mathrm{L})$ were mixed with $990 \mu \mathrm{L}$ of $2 \%$ sodium

411 alginate solution containing $1 \% \mathrm{CaCO}_{3}$ and $50 \mathrm{mM}$ acetate buffer $(\mathrm{pH} 7.0$, Sigma-

412 Aldrich, St. Louis, MO). The mixture was emulsified with $9 \mathrm{~mL}$ of isostearyl alcohol

413 (ISA; Kokyu Alcohol Kogyo Co., Ltd., Chiba, Japan) containing 3\% lecithin (Wako) in 
414 a $50-\mathrm{mL}$ tube by vortexing for $1 \mathrm{~min}$. The emulsion was mixed with $2 \%$ acetic acid in

415 ISA ( $0.1 \mathrm{~mL}$ each) by vortexing for $1 \mathrm{~min}$ with 10 repetitions. During this procedure,

416 the $\mathrm{pH}$ of the mixture gradually decreased to 4.0 and the $\mathrm{CaCO}_{3}$ nanoparticles were

417 dissolved. The released calcium ions from $\mathrm{CaCO}_{3}$ gelated alginate microdroplets in the

418 emulsion. ISA was removed by mixing with $9 \mathrm{~mL}$ of diethyl ether and centrifugation at

$4194^{\circ} \mathrm{C}$ for 3 min using a swing rotor $(3,000 \times \mathrm{g}, 5702 \mathrm{R}$, Eppendorf, Hamburg, Germany),

420 and the residual ISA was further removed from the alginate cores by mixing with 50

$421 \mathrm{mM}$ Tris-HCl buffer ( $\mathrm{pH} 7.5)$ containing $0.2 \%(\mathrm{v} / \mathrm{v})$ Tween 20 (Tris-Tween 20$)(5 \mathrm{~mL})$,

422 followed by centrifugation, and then by repeating the procedure twice with 1-butanol (5

$423 \mathrm{~mL}$ ). The resulting alginate cores were suspended in one volume of $50 \mathrm{mM}$ Tris- $\mathrm{HCl}$

424 (pH 7.5) and filtrated through a 100- $\mu \mathrm{m}$ cell strainer (pluriSelect) to remove large

425 alginate aggregates. The filtrated alginate cores were further washed with Tris- $\mathrm{HCl}(\mathrm{pH}$

426 7.5) and collected in 2-mL microtubes. The volume of the alginate cores was calculated

427 from its weights and stored at $4^{\circ} \mathrm{C}$ after mixing with a one-half volume of Tris- $\mathrm{HCl}(\mathrm{pH}$

428 7.5). To adjust the number of $E$. coli cells in a single alginate core to less than two, 10

$429 \mu \mathrm{L}$ of $3.05 \times 10^{6}-10^{8} E$. coli cells from the $E$. coli stock were mixed with $990 \mu \mathrm{L}$ of the

430 alginate containing $\mathrm{CaCO}_{3}$ suspension. After alginate cores containing E. coli were

431 prepared from the mixture, E. coli in the alginate cores were observed using SYBR 
432 Green I (TaKaRa Bio Inc., Shiga, Japan) (Supplementary Fig. 6). At $3.05 \times 10^{6}$ cell $/ \mathrm{mL}$

433 of the alginate mixture, $17.6 \%$ of the cores contained E. coli and their $78.8 \%$ carried

434 single cells. Thus, $3.05 \times 10^{6}$ cell $/ \mathrm{mL}$ of $E$. coli was used for alginate core preparation in 435 the following experiments.

436

437 Preparation of AGMs containing E. coli cells. The supernatant of the alginate core

438 suspension $(600 \mu \mathrm{L})$ was removed after centrifugation in a $50-\mathrm{mL}$ tube. The residual

439 alginate cores $(400 \mu \mathrm{L})$ were incubated at $35^{\circ} \mathrm{C}$ for 10 min and then mixed with the

440 agarose solution as prepared above $\left(2 \mathrm{~mL}, 35^{\circ} \mathrm{C}\right)$. The mixture was emulsified with

$4410.25 \%(\mathrm{v} / \mathrm{v})$ Span 80 in PGO $\left(20 \mathrm{~mL}, 35^{\circ} \mathrm{C}\right)$ by vortexing for $1 \mathrm{~min}$ (Fig. $\left.1 \mathrm{~b}\right)$. Then, the

442 agarose was gelated by cooling on ice for $1 \mathrm{~h}$. PGO was removed from the emulsion by

443 washing as described above. The alginate gel core was solated by chelating calcium ions

444 with the addition of a 1/10 volume of 0.5 M EDTA (Thermo Fisher Scientific). The

445 AGMs were then suspended in one volume of TE buffer. Large debris in the suspension

446 was removed through a $300 \mu \mathrm{m}$ cell strainer, and the AGMs were further washed with

447 TE. The AGMs were diluted in $50 \mathrm{mM}$ Tris- $\mathrm{HCl}(\mathrm{pH} 7.5)$ containing $0.5 \%$ SeaPlaque

448 agarose, 0.1\% p-phenylene diamine (Wako), and 10,000-fold diluted SYBR Green I

449 (TaKaRa Bio Inc., Shiga, Japan), and the morphology, density, diameter, and number of 
encapsulated E. coli cells were observed under an inverted fluorescence microscope

451 (IX71, Olympus, Tokyo, Japan) equipped with a CCD camera (BU-51LN, Bitran,

452 Saitama, Japan). The AGMs were stored at $4^{\circ} \mathrm{C}$. For long-term storage (more than a 453 week), the AGMs were stored in $40 \%$ ethanol at $-80^{\circ} \mathrm{C}$ and washed three times in 10 454 volumes of water before use.

455

456 Evaluation for alginate core solation in AGM. Alginate gel cores without E. coli

457 were labelled with rhodamine 123 (Wako) using 1-ethyl-3-(3-dimethylaminopropyl)

458 carbodiimide hydrochloride (Tokyo Chemical Industry Co., Ltd., TCI, Tokyo, Japan)

459 and $N$-hydroxysuccinimide (Wako) ${ }^{49}$. AGMs containing rhodamine 123-labelled core

460 were heated at $65^{\circ} \mathrm{C}$ for $5 \mathrm{~min}$ in the presence of $50 \mathrm{mM}$ EDTA or $50 \mathrm{mM} \mathrm{CaCl}_{2}$.

461 Residual cores were observed as mentioned above.

463 Effect of alginate core gelation and solation on MDA. Aliquots $(50 \mu \mathrm{L})$ of the

464 AGMs containing E. coli cells before solation of the alginate cores were mixed with one

465 volume of cell lysis solutions containing $400 \mathrm{mM} \mathrm{KOH}$ with or without $10 \mathrm{mM}$ EDTA

466 and they were neutralised with $100 \mu \mathrm{L}$ of Stop buffer from the REPLI-g UltraFast Mini

467 Kit. The AGMs containing E. coli, AGMs without E. coli (negative control), and 
468 agarose beads containing E. coli (control) were subjected to MDA as described below

469 and their amplified DNA were observed.

470

471 MDA-in-AGM. A $50 \mu \mathrm{L}$ aliquot of AGMs was centrifuged, and the collected AGMs

472 were washed with $200 \mu \mathrm{L}$ water. The cell lysis and DNA denaturation were carried out

473 with Buffer D2 $(50 \mu \mathrm{L})$ of the REPLI-g UltraFast Mini Kit at room temperature for 30

474 min. After denaturation was stopped with the Stop buffer $(50 \mu \mathrm{L})$, the supernatant was

475 removed by centrifugation. The Reaction buffer $(93.5 \mu \mathrm{L})$ containing $11 \mu \mathrm{L}$ phi29 DNA

476 polymerase from the kit was added and incubated at $30^{\circ} \mathrm{C}$ for $3 \mathrm{~h}$ (MDA-in-AGM). The

477 reaction was stopped with 1/10 volume of 0.5 M EDTA, and the AGMs were washed

478 with $200 \mu \mathrm{L}$ of TE buffer three times. The washed AGMs were stored at $4{ }^{\circ} \mathrm{C}$.

479

480 FACS-MDA. Single-cell MDA using a MoFlo XDP fluorescence-activated cell sorter

481 (Beckman Coulter Inc., Brea, CA) was performed as a control ${ }^{38}$. After E. coli, a mock

482 bacterial community, and termite gut bacteria were stained with SYBR Green I or

483 CellTracker Green (Thermo Fisher Scientific), the stained bacteria were sorted into a

484 single cell per well of 96-well PCR plates. The cell lysis was carried out with Buffer D2

$485(1.5 \mu \mathrm{L})$ of the REPLI-g UltraFast Mini Kit at room temperature for $30 \mathrm{~min}$. After 
486 denaturation was stopped with Stop buffer $(1.5 \mu \mathrm{L})$, Reaction buffer $(7.5 \mu \mathrm{L})$ containing

$487 \quad 0.5 \mu \mathrm{L}$ phi29 DNA polymerase from the kit was added and incubated at $30^{\circ} \mathrm{C}$ for $3 \mathrm{~h}$

488 and $65^{\circ} \mathrm{C}$ for $15 \mathrm{~min}$. To check genome amplification and the degree of contamination,

489 the MDA products were evaluated by direct Sanger sequencing of the 16S rRNA. PCR

490 was performed with Bacteria-universal 16S rRNA gene primers: 27F (5'-

491 AGAGTTTGATCMTGGCTCAG) and 1390R (5'-ACGGGCGGTGTGTACAA).

492

493 Mock community of human gut bacteria. A mock community of human gut bacteria

494 was prepared using 15 isolates (Supplementary Table 4) obtained from Japan Collection

495 of Microorganisms. A mixture of all glycerol stocks in equal amounts was used as the

496 mock bacterial community. After the mock bacterial community was diluted 100-fold

497 using water, the diluent $(10 \mu \mathrm{L})$ was treated with DNase $\mathrm{I}(20 \mu \mathrm{L})$ at $30^{\circ} \mathrm{C}$ for $30 \mathrm{~min}$

498 and washed with water $(1 \mathrm{~mL})$ three times. The washed bacterial pellet was suspended

499 in water $(10 \mu \mathrm{L})$. The MDA-in-AGM of the mock bacterial community was prepared

500 from the suspension as mentioned above.

501 The total genomic DNA of the mock bacterial community was extracted using

502 the DNeasy Ultra Clean Microbial Kit (Qiagen). The metagenome sequencing was

503 performed by preparing a library of the purified total genomic DNA with the QIAseq 
504 FX DNA Library Kit (Qiagen). Amplicon sequencing of 16S rRNA genes in the mock

505 community was performed by preparing a library with the Nextera XT Index Kit 96

506 indexes (Illumina, Inc, San Diego, CA) and the MiSeq platform with Reagent Kit V3

507 (600 cycles). The bacterial composition of the mock community was estimated on the

508 basis of the results analysed in QIIME2 ${ }^{50}$.

509

510 Termite gut bacteria. Specimens of the wood-feeding termite Reticulitermes speratus

511 were collected at Tsukuba in Ibaraki Prefecture, Japan. The guts of five worker termites

512 were removed, and the gut contents were suspended in solution $\mathrm{U}$, which consisted of

$5139.2 \mathrm{mM} \mathrm{NaHCO}_{3}, 5.1 \mathrm{mM}$ trisodium citrate dihydrate, $13 \mathrm{mM} \mathrm{KH}_{2} \mathrm{PO}_{4}, 37 \mathrm{mM} \mathrm{NaCl}_{\text {, }}$

$5140.75 \mathrm{mM} \mathrm{CaCl}_{2}$, and $0.4 \mathrm{mM} \mathrm{MgSO}_{4}$. The extracellular DNA and protist DNA were

515 digested using DNase I, and the bacterial cells were collected by centrifugation and

516 washed with water ${ }^{38}$. The washed termite gut bacteria were suspended in water $(10 \mu \mathrm{L})$.

517 The MDA-in-AGM of the termite gut bacteria was prepared using the suspension as

518 mentioned above.

519

520 Genome sequencing and bioinformatics. AGMs containing amplified DNA,

521 detected with SYBR Green I, were transferred individually to PCR tubes, under an 
522 inverted fluorescence microscope equipped with a micromanipulator (TransferMan

523 NK2, Eppendorf, Hamburg, Germany). Water $(29 \mu \mathrm{L})$ was added to each PCR tube

524 with a single AGM and incubated at $60^{\circ} \mathrm{C}$ for $5 \mathrm{~min}$, to release DNA by solating the

525 agarose shell. To check the degree of contamination, we screened the MDA-in-AGM

526 products by the direct Sanger sequencing of the 16S rRNA as mentioned above. After

527 removing the contaminated samples, sequencing libraries were prepared using the

528 QIAseq FX DNA Library Kit. When the sequencing libraries were constructed using

529 adaptors with the concentration described in the manufacturer's instructions, a large

530 number of concatenated adaptor sequences were formed. To suppress the generation of

531 the artificial sequences, the concentration of adaptor sequences was adjusted to one-

532 tenth of that in the manufacturer's instructions. Genome sequencing was performed on

533 the MiSeq platform with Reagent Kit V3 (600 cycles). Sequence libraries for single

534 cells isolated from the termite gut microbiota using FACS were prepared using the

535 Nextera XT DNA Library Prep Kit (Illumina), and analysed on the Illumina HiSeq 2500

536 platform (500 cycles).

537 The generated short reads were trimmed using Cutadapt

538 (https://github.com/marcelm/cutadapt), PRINSEQ (http://prinseq.sourceforge.net/),

539 FASTX Trimmer, FASTQ Quality Trimmer 
540 (http://hannonlab.cshl.edu/fastx_toolkit/download.html), and cmpfastq pe

541 (http://compbio.brc.iop.kcl.ac.uk/software/download/cmpfastq_pe). The trimmed reads

542 were assembled using SPAdes ver. 3.13 .0 (k-mer: 21, 33, 55, 77, 99, and 127, options: -

543 -sc, --careful) $)^{31}$ into contigs. Only contigs $>1 \mathrm{~kb}$ were selected using SeqKit

544 (https://bioinf.shenwei.me/seqkit/) for the subsequent analyses. The fixed number of

545 read pairs was randomly chosen by SeqKit. Taxonomic classification of single-cell

546 genomes from termite gut samples was conducted using the Genome Taxonomy

547 Database Tool Kit (GTDB-Tk) $)^{39}$. Genomic sequence data showing $>5 \%$ contamination,

548 identified by using CheckM ${ }^{32}$, were sequentially subjected to binning, refinement, and

549 reassembly processes with the BINNING (including metaBAT2 ${ }^{51}, \operatorname{MaxBin}^{52}$, and

550 CONCOCT ${ }^{53}$ ), BIN_REFINEMENT, and REASSEMBLE_BINS modules of

551 metaWRAP ${ }^{34}$. For the single-cell genome analyses using E. coli DH5 $\alpha$ and the human

552 gut bacteria, trimmed reads were mapped onto known genome sequences using

553 Bowtie $2^{54}$, and the results were visualised using IGV ver. 2.3.26

554 (http://software.broadinstitute.org/software/igv/). The genome coverage obtained by

555 mapping reads without assembling them [total length (base) of mapped reads divided by

556 reference genome size] was calculated using BBtools (http://jgi.doe.gov/data-and-

557 tools/bbtools/). The chimeric read rate was calculated by mapping short reads to the 
558 reference genome with the Barrows-Wheeler Aligner

559 (https://sourceforge.net/projects/bio-bwa/files/) and SAMtools (http://www.htslib.org).

560 The reference genomes are listed in Supplementary Table 4. Cross-contaminations of

561 genomic sequencing data with $<5 \%$ contamination using CheckM $^{32}$ (Supplementary

562 Table 7) were calculated by mapping sequencing reads to reference genomes in the

563 mock community ${ }^{17}$.

564

565

566 Acknowledgements

567 This work was supported by the RIKEN Pioneering Project "Biology of Symbiosis",

568 JSPS KAKENHI Grants 16K07224 to H. A, and 17H01447 and 19H05679 to M. O.,

569 and Institute of Fermentation, Osaka to M. Y.

570

571 Author contributions

572 Y. Y. and M. O. supervised the study. H. A., M. Y., Y. H., and M. O. designed the

573 study. H. A., M. Y., and Y. H. wrote the manuscript. H. A. elucidated the AGM

574 preparation protocol. M. Y. and M. S. performed next genome sequencings and their

575 data analyses. All authors contributed to and edited the manuscript. 
577 Data availability

578 The raw fastq files (DRR252532-DRR253885) and assemblies for SAGs of termite gut

579 bacteria (BNTM01000000-BOCE01000000) were deposited into DDBJ under

580 BioProject accession no. PRJDB10679.

581

582 Competing interests statement

583 The authors have no conflicts of interest to declare.

584

585 References

586 1. Woyke, T., Doud, D. F. R. \& Schulz, F. The trajectory of microbial single-cell

587 sequencing. Nat. Methods 14, 1045-1054 (2017).

588 2. Wrighton, K. C. et al. Fermentation, Hydrogen, and Sulfur Metabolism in

589 Multiple Uncultivated Bacterial Phyla. Science 337, 1661-1665 (2012).

590 3. Albertsen, M. et al. Genome sequences of rare, uncultured bacteria obtained by

591 differential coverage binning of multiple metagenomes. Nat. Biotechnol. 31, 533-538

592 (2013). 
593 4. Anantharaman, K. et al. Thousands of microbial genomes shed light on

594 interconnected biogeochemical processes in an aquifer system. Nat. Commun. 7, 1-11

595 (2016).

596 5. Delmont, T. O. et al. Nitrogen-fixing populations of Planctomycetes and

597 Proteobacteria are abundant in surface ocean metagenomes. Nat. Microbiol. 3, 804-813

598 (2018).

$599 \quad 6 . \quad$ Zhang, K. et al. Sequencing genomes from single cells by polymerase cloning.

600 Nat. Biotechnol. 24, 680-686 (2006).

6017 Kaster, A.-K. \& Sobol, M. S. Microbial single-cell omics: the crux of the

602 matter. Appl. Microbiol. Biotechnol. 104, 8209-8220 (2020).

603 8. Woyke, T. et al. One Bacterial Cell, One Complete Genome. PLoS One 5,

604 e10314 (2010).

605 9. Fu, A. Y., Spence, C., Scherer, A., Arnold, F. H. \& Quake, S. R. A

606 microfabricated fluorescence-activated cell sorter. Nat. Biotechnol. 17, 1109-1111

607 (1999).

608 10. Marcy, Y. et al. Nanoliter reactors improve multiple displacement

609 amplification of genomes from single cells. PLoS Genet. 3, 1702-1708 (2007). 
610 11. Joensson, H. N. \& Andersson Svahn, H. Droplet Microfluidics-A Tool for

611 Single-Cell Analysis. Angew. Chem. Int. Ed. 51, 12176-12192 (2012).

612 12. Zong, C., Lu, S., Chapman, A. R. \& Xie, X. S. Genome-Wide Detection of

613 Single-Nucleotide and Copy-Number Variations of a Single Human Cell. Science 338,

$614 \quad 1622-1626(2012)$

615 13. Dean, F. B. et al. Comprehensive human genome amplification using multiple

616 displacement amplification. Proc. Natl. Acad. Sci. USA 99, 5261-5266 (2002).

617 14. Alneberg, J. et al. Genomes from uncultivated prokaryotes: a comparison of

618 metagenome-assembled and single-amplified genomes. Microbiome 6, 173 (2018).

619 15. Stepanauskas, R. et al. Improved genome recovery and integrated cell-size

620 analyses of individual uncultured microbial cells and viral particles. Nat. Commun. 8,

$62184(2017)$

622 16. Gole, J. et al. Massively parallel polymerase cloning and genome sequencing

623 of single cells using nanoliter microwells. Nat. Biotechnol. 31, 1126-1132 (2013).

624 17. Xu, L., Brito, I. L., Alm, E. J. \& Blainey, P. C. Virtual microfluidics for digital 625 quantification and single-cell sequencing. Nat. Methods 13, 759-762 (2016). 
626 18. Sidore, A. M., Lan, F., Lim, S. W. \& Abate, A. R. Enhanced sequencing

627 coverage with digital droplet multiple displacement amplification. Nucleic Acids Res.

628 44, e66 (2016).

629 19. Nishikawa, Y. et al. Monodisperse Picoliter Droplets for Low-Bias and

630 Contamination-Free Reactions in Single-Cell Whole Genome Amplification. PLoS One

631 10, e0138733 (2015).

632 20. Hosokawa, M., Nishikawa, Y., Kogawa, M. \& Takeyama, H. Massively

633 parallel whole genome amplification for single-cell sequencing using droplet

634 microfluidics. Sci. Rep. 7, 5199 (2017).

635 21. Chijiiwa, R. et al. Single-cell genomics of uncultured bacteria reveals dietary 636 fiber responders in the mouse gut microbiota. Microbiome 8, 5 (2020).

637 22. Hammond, M., Homa, F., Andersson-Svahn, H., Ettema, T. J. G. \& Joensson,

638 H. N. Picodroplet partitioned whole genome amplification of low biomass samples

639 preserves genomic diversity for metagenomic analysis. Microbiome 4, 52 (2016).

640 23. Bigdeli, S., Dettloff, R. O., Frank, C. W., Davis, R. W. \& Crosby, L. D. A

641 simple method for encapsulating single cells in alginate microspheres allows for direct

642 PCR and whole genome amplification. PLoS One 10, e0117738 (2015). 
643 24. Lan, F., Demaree, B., Ahmed, N. \& Abate, A. R. Single-cell genome

644 sequencing at ultra-high-throughput with microfluidic droplet barcoding. Nat.

645 Biotechnol. 35, 640-646 (2017).

646 25. Rabanel, J. M., Banquy, X., Zouaoui, H., Mokhtar, M. \& Hildgen, P. Progress

647 technology in microencapsulation methods for cell therapy. Biotechnol. Prog. 25, 946-

$648963(2009)$.

649 26. Siltanen, C. et al. One step fabrication of hydrogel microcapsules with hollow

650 core for assembly and cultivation of hepatocyte spheroids. Acta Biomater. 50, 428-436

651 (2017).

652 27. Breguet, V., Gugerli, R., von, S. U. \& Marison, I. W. CHO immobilization in

653 alginate/poly-L-lysine microcapsules: an understanding of potential and limitations.

654 Cytotechnology 53, 81-93 (2007).

655 28. Sakai, S., Hashimoto, I. \& Kawakami, K. Production of cell-enclosing hollow-

656 core agarose microcapsules via jetting in water-immiscible liquid paraffin and formation

657 of embryoid body-like spherical tissues from mouse ES cells enclosed within these

658 microcapsules. Biotechnol. Bioeng. 99, 235-243 (2008). 
659 29. Tamminen, M. V. \& Virta, M. P. J. Single gene-based distinction of individual

660 microbial genomes from a mixed population of microbial cells. Front. Microbiol. 6,

661 (2015).

662 30. Poncelet, D. Production of alginate beads by emulsification/internal gelation.

663 Ann. N. Y. Acad. Sci. 944, 74-82 (2001).

664 31. Bankevich, A. et al. SPAdes: A New Genome Assembly Algorithm and Its

665 Applications to Single-Cell Sequencing. J. Comput. Biol. 19, 455-477 (2012).

666 32. Parks, D. H., Imelfort, M., Skennerton, C. T., Hugenholtz, P. \& Tyson, G. W.

667 CheckM: assessing the quality of microbial genomes recovered from isolates, single

668 cells, and metagenomes. Genome Res. 25, 1043-1055 (2015).

669 33. Gurevich, A., Saveliev, V., Vyahhi, N. \& Tesler, G. QUAST: quality

670 assessment tool for genome assemblies. Bioinformatics 29, 1072-1075 (2013).

671 34. Uritskiy, G. V., DiRuggiero, J. \& Taylor, J. MetaWRAP-a flexible pipeline for

672 genome-resolved metagenomic data analysis. Microbiome 6, 158 (2018).

673 35. Lasken, R. S. \& Stockwell, T. B. Mechanism of chimera formation during the

674 Multiple Displacement Amplification reaction. BMC Biotechnol. 7, 19 (2007). 
675 36. Tu, J. et al. Systematic Characteristic Exploration of the Chimeras Generated

676 in Multiple Displacement Amplification through Next Generation Sequencing Data

677 Reanalysis. PLoS One 10, e0139857 (2015).

678 37. Hongoh, Y., Ohkuma, M. \& Kudo, T. Molecular analysis of bacterial

679 microbiota in the gut of the termite Reticulitermes speratus (Isoptera; Rhinotermitidae).

680 FEMS Microbiol. Ecol. 44, 231-242 (2003).

681 38. Yuki, M. et al. Dominant ectosymbiotic bacteria of cellulolytic protists in the

682 termite gut also have the potential to digest lignocellulose. Environ. Microbiol. 17,

$683 \quad 4942-4953(2015)$.

684 39. Chaumeil, P. A., Mussig, A. J., Hugenholtz, P. \& Parks, D. H. GTDB-Tk: a

685 toolkit to classify genomes with the Genome Taxonomy Database. Bioinformatics

686 (2019).

687 40. Hongoh, Y. et al. Complete genome of the uncultured Termite Group 1

688 bacteria in a single host protist cell. Proc Natl Acad Sci U A 105, 5555-5560 (2008).

689 41. Izawa, K. et al. Comparison of Intracellular "Ca. Endomicrobium

690 Trichonymphae" Genomovars Illuminates the Requirement and Decay of Defense

691 Systems against Foreign DNA. Genome Biol. Evol. 8, 3099-3107 (2016). 
692 42. Leonaviciene, G., Leonavicius, K., Meskys, R. \& Mazutis, L. Multi-step

693 processing of single cells using semi-permeable capsules. Lab. Chip 20, 4052-4062

694 (2020).

695 43. Rodrigue, S. et al. Whole Genome Amplification and De novo Assembly of

696 Single Bacterial Cells. PLoS One 4, e6864 (2009).

697 44. Zheng, G. X. et al. Massively parallel digital transcriptional profiling of single

698 cells. Nat. Commun. 8, 14049 (2017).

699 45. Clingenpeel, S., Clum, A., Schwientek, P., Rinke, C. \& Woyke, T.

700 Reconstructing each cell's genome within complex microbial communities-dream or

701 reality? Front. Microbiol. 5, 771 (2015).

702 46. Cadet, J., Douki, T. \& Ravanat, J.-L. Oxidatively Generated Damage to

703 Cellular DNA by UVB and UVA Radiation,. Photochem. Photobiol. 91, 140-155

704 (2015).

705 47. Nayfach, S., Shi, Z. J., Seshadri, R., Pollard, K. S. \& Kyrpides, N. C. New

706 insights from uncultivated genomes of the global human gut microbiome. Nature 568,

$707 \quad 505-510(2019)$. 
708 48. Merino, N. et al. Single-Cell Genomics of Novel Actinobacteria With the

709 Wood-Ljungdahl Pathway Discovered in a Serpentinizing System. Front. Microbiol.

710 11, e1031 (2020).

711 49. Hermanson, G. T. Bioconjugate Techniques. (Academic Press, San Diego, 712 1996).

713 50. Bolyen, E. et al. Reproducible, interactive, scalable and extensible microbiome

714 data science using QIIME 2. Nat. Biotechnol. 37, 852-857 (2019).

715 51. Kang, D. D. et al. MetaBAT 2: an adaptive binning algorithm for robust and

716 efficient genome reconstruction from metagenome assemblies. PeerJ 7, e7359 (2019).

717 52. Wu, Y.-W., Simmons, B. A. \& Singer, S. W. MaxBin 2.0: an automated

718 binning algorithm to recover genomes from multiple metagenomic datasets.

719 Bioinformatics 32, 605-607 (2016).

720 53. Alneberg, J. et al. Binning metagenomic contigs by coverage and composition.

$721 \quad$ Nat. Methods 11, 1144-1146 (2014).

722 54. Langmead, B. \& Salzberg, S. L. Fast gapped-read alignment with Bowtie 2.

723 Nat. Methods 9, 357-359 (2012). 
725 Figure legends

726 Fig. 1. Schematic view of an agarose gel microcapsule (AGM) and its 727 preparation process.

728 a. A schematic diagram of the AGM developed in this study for the single-cell isolation

729 and multiple displacement amplification (MDA). The AGM consists of an agarose

730 hydrogel shell and an alginate sol core. The alginate core provides a picolitre-scale

731 reaction chamber for MDA, while the agarose shell acts both as a permeable envelope

732 for enzymes and small molecules and as a protective wall against larger particles and

733 molecules such as bacterial cells and genomic/amplified DNA.

734 b. A preparation scheme for AGMs containing Escherichia coli cell(s). The alginate

735 cores and agarose shells are gelated with $\mathrm{Ca}^{2+}$ from $\mathrm{CaCO}_{3}$ and cooled in emulsion,

736 respectively. PGO (polyglyceryl-6 octacaprylate) can suppress the aggregation of

737 AGMs during agarose gelation by cooling. Finally, the alginate gel cores are solated

738 with EDTA by chelating $\mathrm{Ca}^{2+}$. ISA: isostearyl alcohol.

739

740 Fig. 2. Yield enhancement of multiple displacement amplification (MDA) by 

alginate core solation.

742 The effect of alginate core solation on the productivity of MDA within agarose gel

743 microcapsules (AGMs) was evaluated. The genomic DNA of Escherichia coli

744 encapsulated within AGMs was amplified by MDA with or without alginate solation. A

745 phase contrast image (red) and epifluorescent image (green, SYBR Green I) are

746 overlaid. MDA in agarose gel beads (without an alginate core) was also conducted as a

747 control experiment. Arrows indicate E. coli cells stained with SYBR Green I. Bar $=100$ $748 \mu \mathrm{m}$.

750 Fig. 3. Comparison of genome completeness and amplification bias of single-

751 cell genomes obtained by MDA-in-AGM and FACS-MDA.

752 a. Completeness and number of contigs of SAGs during the sequencing process. Box-

753 and-violin plots (blue: MDA-in-AGM; red: FACS-MDA) are shown for Escherichia

754 coli and two bacterial species as examples from the mock community of human gut

755 bacteria (Supplementary Tables 2, 5, and 6). Data points (blue circle) and their

756 arithmetic means (rhombus) are shown on the plots. In the E. coli graphs, the results for

757 DNA prepared from cultured E. coli cells without MDA are also shown (green circle). 
758 In the left panels, significant differences are indicated with asterisks $(P<0.05)$ or

759 double asterisks $(P<0.01)$.

760 b. The number of sequence reads mapped against genome regions is shown as an

761 indicator of amplification bias caused during MDA. The number of reads $\left(\times 10^{5}\right)$ used

762 for de novo assembly (shown as 'Reads') and genome completeness (\%) ('Compl.')

763 estimated using CheckM are shown.

765 Fig. 4. Relationships between genome completeness and GC contents of single-

766 cell genomes in the mock community of human gut bacteria.

767 Results were obtained by assembling randomly chosen $3 \times 10^{5}$ read pairs for each

768 single-cell amplified genome. MS485-1-51 of MDA-in-AGM $\left(2.6 \times 10^{5}\right.$ read pairs $)$ and

769 CS1-94 of FACS-MDA $\left(2.9 \times 10^{5}\right.$ read pairs $)$ were assembled using all read pairs. The

770 bacterial species comprising the mock community and detailed results are shown in

771 Supplementary Tables 4-6. Blue and red indicate the MDA-in-AGM and the FACS-

772 MDA, respectively. Squares, circles, and rhombuses indicate gram-positive, gramnegative, and gram-variable bacteria, respectively. 


\begin{tabular}{|c|c|c|c|c|c|c|}
\hline \multirow[b]{2}{*}{ Taxon of bacteria } & \multicolumn{3}{|c|}{ MDA-in-AGM } & \multicolumn{3}{|c|}{ FACS-MDA $^{\dagger}$} \\
\hline & $\begin{array}{l}\text { Number } \\
\text { of SAGs }\end{array}$ & $\begin{array}{c}\text { Genome } \\
\text { complete- } \\
\text { ness }(\%)\end{array}$ & $\begin{array}{l}\text { Contami- } \\
\text { nation }(\%)\end{array}$ & $\begin{array}{l}\text { Number } \\
\text { of SAGs }\end{array}$ & $\begin{array}{c}\text { Genome } \\
\text { complete- } \\
\text { ness }(\%)\end{array}$ & $\begin{array}{l}\text { Contami- } \\
\text { nation }(\%)\end{array}$ \\
\hline Spirochaetia & 15 & $73.5 \pm 20.6$ & $0.9 \pm 1.1$ & 35 & $32.5 \pm 19.0$ & $0.3 \pm 0.6$ \\
\hline Bacteroidia & 8 & $88.2 \pm 8.7$ & $1.3 \pm 0.7$ & 51 & $36.1 \pm 17.7$ & $0.6 \pm 1.2$ \\
\hline Alphaproteobacteria & 2 & $87.0 \pm 1.3$ & $0.0 \pm 0.0$ & 8 & $52.9 \pm 19.5$ & $0.9 \pm 1.7$ \\
\hline Betaproteobacteria & 7 & $87.5 \pm 5.9$ & $1.2 \pm 0.3$ & 9 & $35.3 \pm 12.6$ & $0.9 \pm 0.8$ \\
\hline Deltaproteobacteria & 3 & $86.4 \pm 6.7$ & $0.5 \pm 0.3$ & 3 & $22.8 \pm 16.7$ & $1.1 \pm 0.6$ \\
\hline Epsilonproteobacteria & 2 & $55.0 \pm 48.5$ & $2.2 \pm 3.1$ & 2 & $38.8 \pm 20.7$ & $1.2 \pm 0.6$ \\
\hline Clostridia & 4 & $70.2 \pm 23.6$ & $1.3 \pm 0.4$ & 27 & $36.7 \pm 16.2$ & $0.7 \pm 1.1$ \\
\hline Actinobacteria & 1 & 51.9 & 0.87 & 3 & $33.5 \pm 15.9$ & $1.9 \pm 1.7$ \\
\hline Planctomycetales & 1 & 87.1 & 0.1 & 11 & $42.6 \pm 21.0$ & $0.6 \pm 0.8$ \\
\hline Synergistia & 2 & $83.9 \pm 22.7$ & $1.2 \pm 1.6$ & 4 & $65.5 \pm 21.9$ & $0.2 \pm 0.3$ \\
\hline Fibrobacteria & 1 & 72.8 & 0.0 & n. d. & n. d. & n. d. \\
\hline Deferribacteres & 1 & 72.9 & 2.0 & n. d. & n. d. & n. d. \\
\hline $\begin{array}{l}\text { Candidate division } \\
\text { SR1 }\end{array}$ & 1 & 74.1 & 0.0 & n. d. & n. d. & n. d. \\
\hline Bacilli & n. d. & n. d. & n. d. & 8 & $47.2 \pm 23.2$ & $1.4 \pm 2.0$ \\
\hline Endomicrobia $^{*}$ & 18 & $91.2 \pm 9.8$ & $1.5 \pm 1.0$ & n. t. & n. t. & n. t. \\
\hline $\begin{array}{l}\text { Total sequenced } \\
\text { samples }\end{array}$ & $48(66)$ & $\begin{array}{c}78.6 \pm 18.2 \\
(82.0 \pm 17.2)\end{array}$ & $\begin{array}{c}1.0 \pm 1.0 \\
(1.1 \pm 1.0)\end{array}$ & 161 & $37.7 \pm 19.0$ & $0.7 \pm 1.1$ \\
\hline
\end{tabular}

778 Table 1 Summary of single-cell amplified genomes (SAGs) of symbiotic

779 bacteria in termite gut bacteria.

780 n. d.: not detected. n. t.: not tested.

$781 \dagger$ NGS libraries were made by Nextera XT and read by HiSeq.

782 Number of SAGs, genome completeness, and contamination including Endomicrobia

783 are shown in parentheses. 
786 Figures

787

a

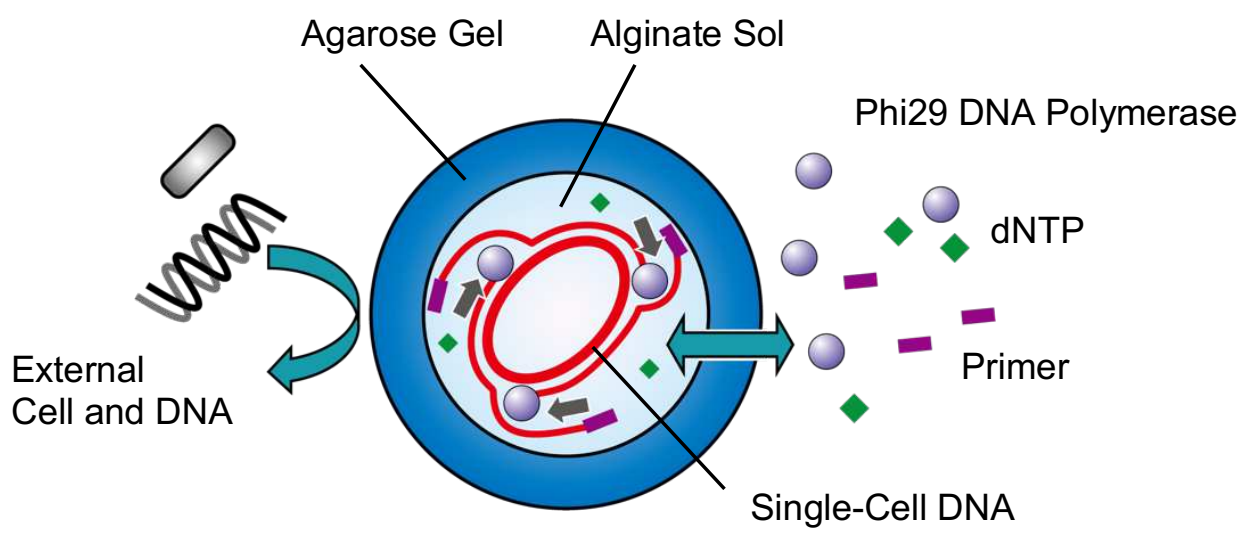

b
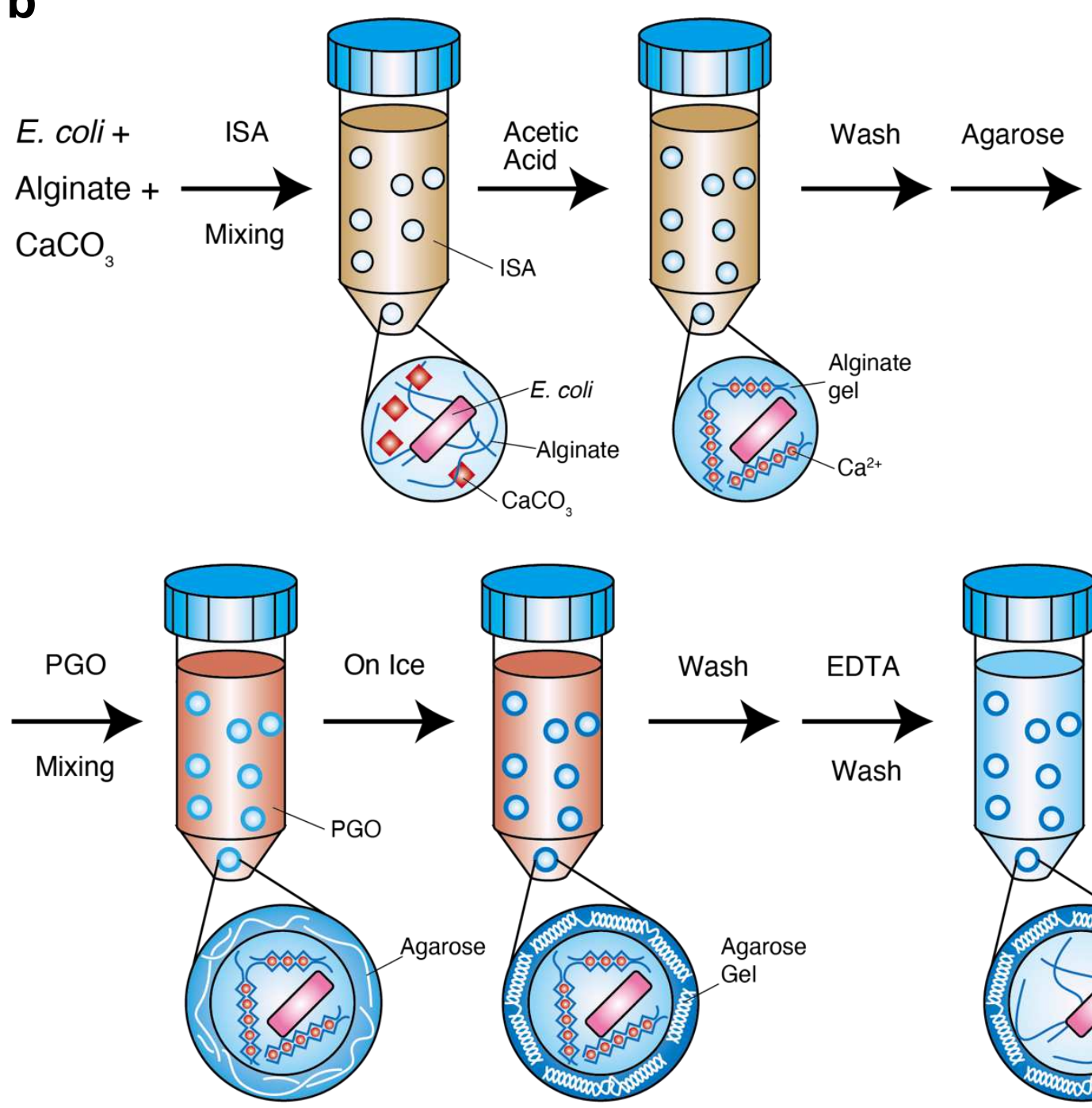

Fig. 1

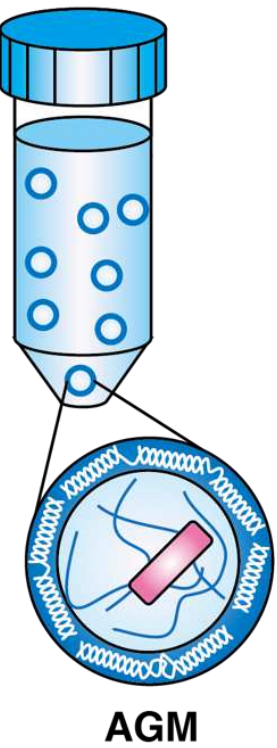




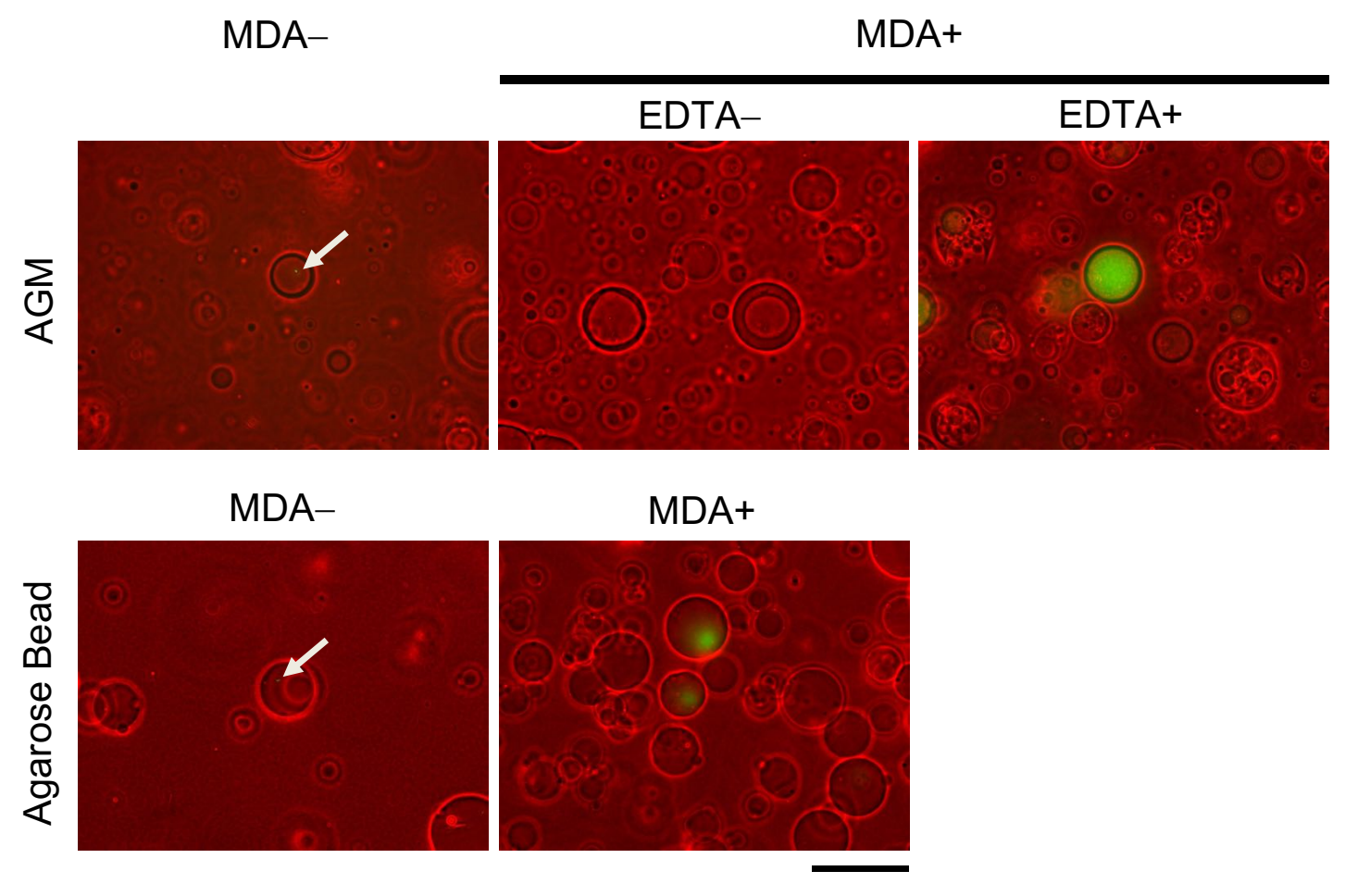

Fig. 2 


\section{a}

Escherichia coli

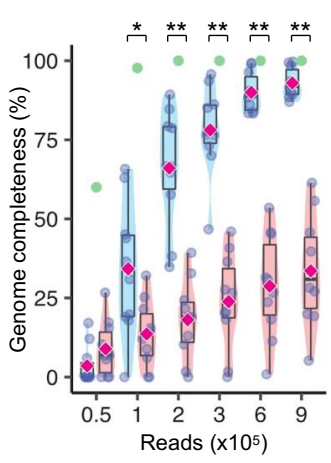

Bacteroides thetaiotaomicron

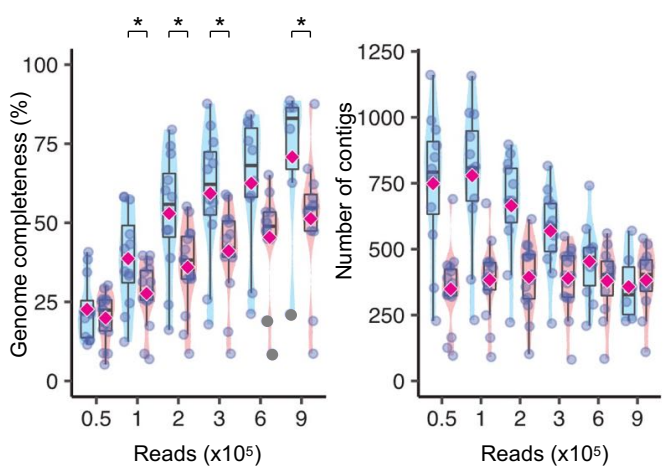

Parabacteroides distasonis

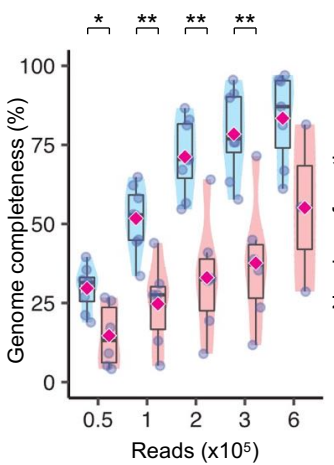

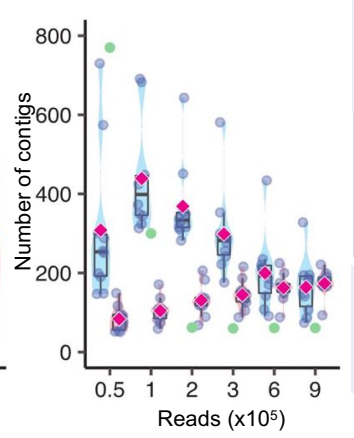

b

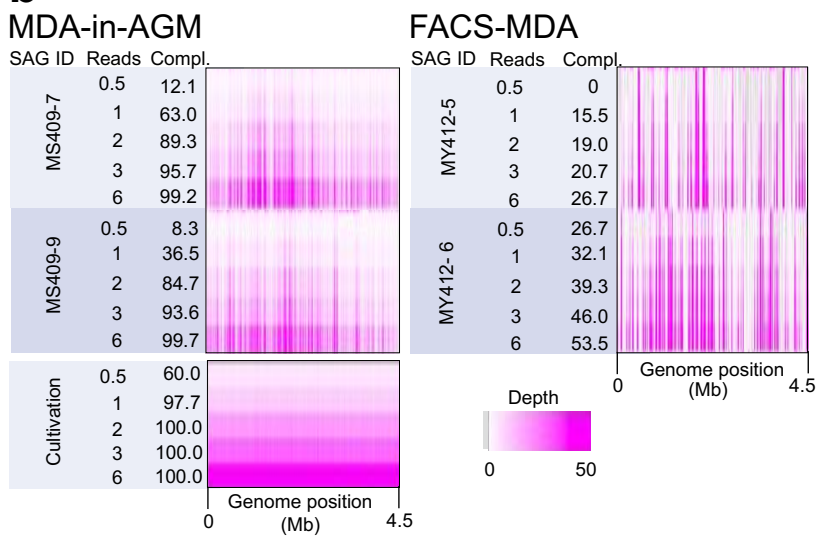

MDA-in-AGM

FACS-MDA

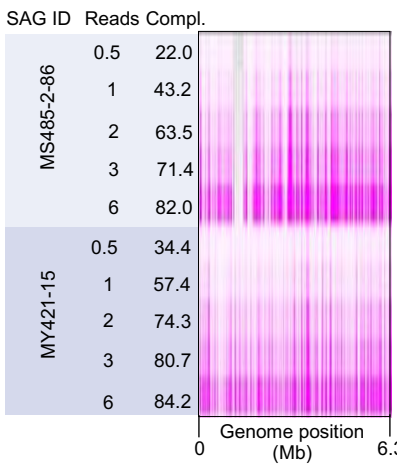

SAG ID Reads Compl.

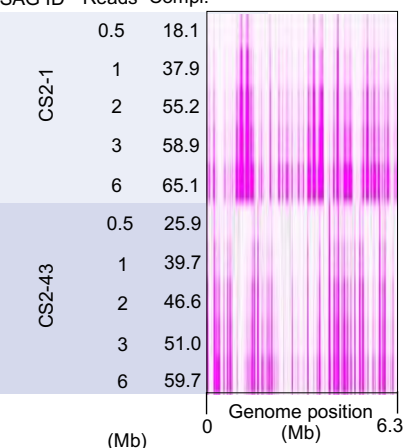

(Mb)

\section{FACS-MDA}

SAG ID Reads Compl.

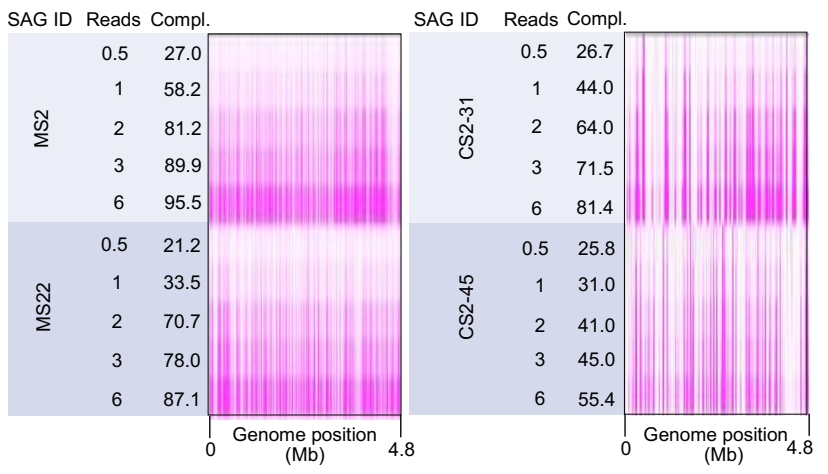

Fig. 3 


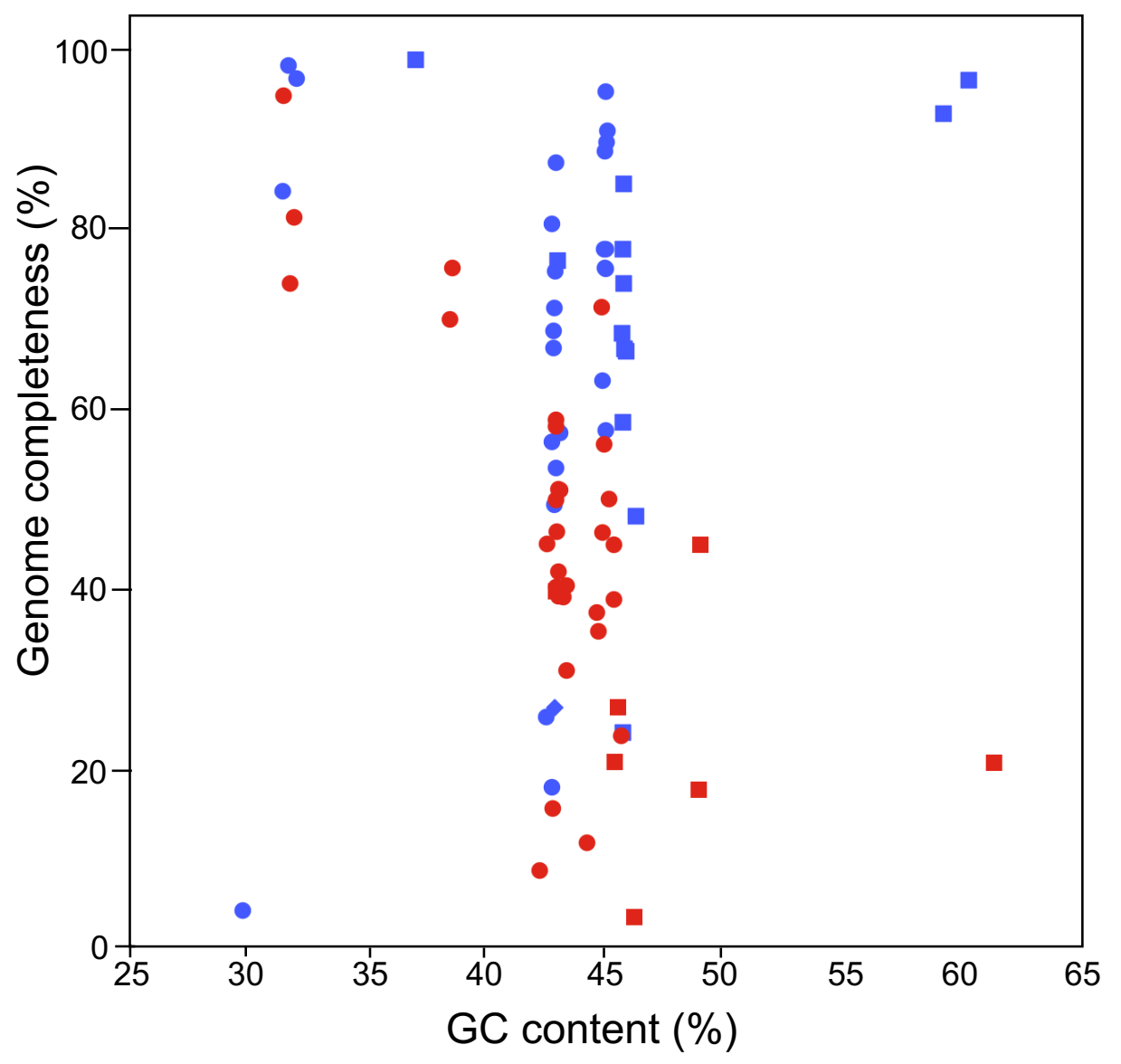

Fig. 4 


\section{Supplementary Files}

This is a list of supplementary files associated with this preprint. Click to download.

- 220119SingleCelIMDAinAGMSciRepSI300dpi.pdf 